\title{
PREDICTED SINGLE POINT MUTATIONS IN GANGLIOSIDE-BINDING DOMAIN OF SARS-COV-2 S AND RECOGNITION BY 9-O-ACETYLATED SIALIC ACID AND HIDROXYCHLOROQUINE
}

\author{
Petar M. MITRASINOVIC \\ Center for Biophysical and Chemical Research, Belgrade Institute of Science and Technology, 11060 Belgrade, Serbia
}

Received September 14, 2020

\begin{abstract}
The infectious disease CoViD-19 is caused by a new severe acute respiratory syndromecoronavirus-2 (SARS-CoV-2). A possible infection mechanism includes dual host receptor recognitions by the SARS-CoV-2 transmembrane spike (S) glycoproteins. SARS-CoV-2 S contains two different domains, the receptor-binding domain (RBD) and the N-terminal domain (NTD), which interact with angiotensin-converting enzyme 2 (ACE2) and the ganglioside-rich domain of the plasma membrane at the surface of respiratory cell, respectively. NTD amino acids (111-162) form a functional ganglioside-binding domain (GBD) that is conserved in all clinical isolates. Herein, the single point mutations (SPMs) of GBD residues to which the virus is prone during genetic adaptation are predicted using an in silico protein engineering approach. Consequently, their effects on the attachment of SARS-CoV-2 $\mathrm{S}$ to the ganglioside-linked 9-O-acetylated sialic acid (9-O-Ac-Sia) are explored using molecular docking simulations. Val120Tyr and Asn122Trp are found to be the most likely SPMs in the GBD of SARS-CoV-2 S being involved in very specific recognition with 9-O-Ac-Sia through electrostatic interactions. Val120Tyr and Asn122Trp are also found to be the most likely SPMs in the GBD of SARS-CoV-2 S that is involved in conspicuously hydrophobic recognition with hidroxychloroquine (Hcq), thereby indicating the ability of Hcq to competitively inhibit GBD interactions with lipid rafts. However, the considerably non-specific binding of $\mathrm{Hcq}$ and the micromolar range of the dissociation constants of the SARS-CoV-2 S/Hcq complexes do not support the proposal of treating Hcq as a drug candidate. A usable guideline for the structure-based design of anti-CoViD19 drugs is given.
\end{abstract}

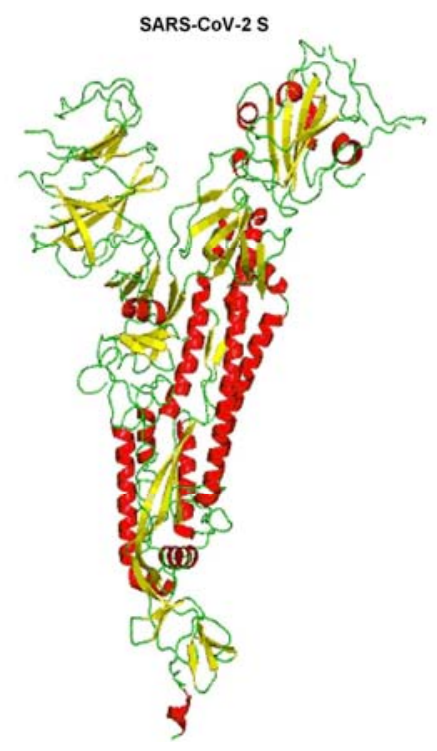

\section{INTRODUCTION}

Coronaviruses (CoVs) are involved in respiratory, enteric, hepatic and neuronal infectious diseases in animals and in humans. The beta genus of the enveloped, single-stranded RNA (+) viruses comprises bCoV, hCoV-OC43, MHV, SARS-CoV

\footnotetext{
i WHO Director-General's Opening Remarks at the Media Briefing on CoViD-19. https://www.who.int/dg/speeches/ detail/who-director-general-s-opening-remarks-at-the-mediabriefing-on-covid-19---11-march-2020 (accessed 7 February 2021).
}

and MERS-CoV. ${ }^{1}$ The new virus SARS-CoV-2, also referred to as $\mathrm{hCoV}-19$, caused an infectious disease CoViD-19 in 2019 in Wuhan, China. ${ }^{2}$ Since then CoViD-19 has spread through interpersonal contacts worldwide. The World Health Organization (WHO) declared a world 2019-20 CoV pandemic on March 11, 2020. Substantial efforts are being invested through current research and therapeutic product development aiming at mitigating the far-reaching consequences of the pandemic on human lives. An adequate vaccine is expected to be available on the

* Corresponding author: pmitrasinovic.ist-belgrade.edu.rs@tech-center.com 
market in the foreseeable future. ${ }^{\text {ii }}$ The US Food and Drug Administration (FDA) has been working on a variety of coronavirus treatments, such as chloroquine, hidroxychloroquine and plasma from recovered CoViD-19 patients. ${ }^{\text {iii }}$ As an initial therapy, Hcq was suggested to be more preferred than $\mathrm{Cq}^{3}$ As a less toxic derivative of $\mathrm{Cq}$, Hcq was supposed to be effective in inhibiting the SARS$\mathrm{CoV}-2$ infection in vitro. ${ }^{4}$ Even though $\mathrm{Hcq}$ was shown to have decent binding affinity toward the SARS-CoV-2 S-ACE2 complex, ${ }^{5,6}$ Hcq can cause retinopathy that is dose and time dependent, as well as less likely cardiac and auditory toxicity, meaning that its adverse effects should be diagnosed and prevented on time. ${ }^{7,8}$ The FDA decided to cancel its initial emergency use authorization for $\mathrm{Cq}$ and $\mathrm{Hcq}$, as Hcq worked no better than a placebo in preventing CoViD-19 infections. ${ }^{9}$ Consequently, the WHO also decided to drop Hcq from its global study of potential treatments for CoViD-19. . ${ }^{\text {iv }}$ The present work is intended to provide some new insights into these standpoints at the molecular scale.

Infections are mediated by the recognition between the SARS-CoV-2 $\mathrm{S}$ proteins and the ACE2 receptors at cell surface. SARS-CoV-2 S binds ACE2 with larger affinity than does SARSCoV S. ${ }^{10}$ SARS-CoV-2 S contains two distinct domains (Fig. 1, top, left), the receptor-binding domain (RBD) and the N-terminal domain (NTD), interacting with ACE2 and the ganglioside-rich domain of the plasma membrane, respectively. ${ }^{3}$ The amino acid residues (111-162) of NTD form GBD (Fig. 1, top, right) - a functional domain being both involved in facilitating the ACE2 recognition by RBD and completely conserved in clinical isolates worldwide. The outline of this article is to place specificity issue, underlying the recognition of ganglioside by $\mathrm{GBD}$, on a more rational ground.

Based on the structural knowledge of SARS$\mathrm{CoV}-2 \mathrm{~S},{ }^{10}$ the recognition modes of GBD by $9-\mathrm{O}-$ Ac-Sia and Hcq are, herein, investigated using molecular docking simulations, primarily in terms of affinity and specificity. Recent in vitro data ${ }^{11}$

\footnotetext{
ii Pharma Chiefs Expect Coronavirus Vaccine in 12-18 Months. https://medicalxpress.com/news/2020-03-pharmachiefs-coronavirus-vaccine-.html (accessed 7 February 2021). iii FDA Testing Coronavirus Treatments, Including Chloroquine, Plasma from Recovered CoViD-19 Patients. https://techcrunch.com/2020/03/19/fda-testing-coronavirustreatments-including-chloroquine-plasma-from-recoveredcovid-19-patients/ (accessed 7 February 2021).

iv FDA Cancels Emergency Approval for Hydroxychloroquine. https://www.labroots.com/trending/ drug-discovery-and-development/17914/fda-cancelshydroxychloroquine (accessed 7 February 2021).
}

highlighted the observation that SARS-CoV-2 mutations, including several ones in the spike glycoprotein, ${ }^{11,12}$ are not an infrequent event and that certain SARS-CoV-2 mutations confer a greater pathogenic effect than others. ${ }^{11}$ In this study, likely SPMs in the GBD of SARS-CoV-2 S are predicted using an in silico protein engineering approach $^{13-16}$ in a systematic fashion. Consequently, a question, how the most likely SPMs affect the affinity and specificity of 9-O-AcSia and Heq to GBD, is explored.

\section{EXPERIMENTAL}

The atomic coordinates of the receptor were obtained by retrieving the experimental structure of the trimeric SARSCoV-2 S (PDB ID: 6VSB) from the Research Collaboratory for Structural Bioinformatics (RCSB) Protein Data Bank (PDB). ${ }^{10}$

The FoldX 5.0 suite of programs ${ }^{17-25}$ was systematically exploited to generate the single point mutations of GBD residues. The protocol uses full atomic descriptions of the structures of the proteins, with different energy terms being parameterized using empirical data from protein engineering experiments. The predictive power was tested on a very large set of point mutants (1088) that comprise most of the structural environments found in proteins. ${ }^{26}$ The single point mutations were predicted at $\mathrm{pH} 8-\mathrm{a}$ value used for the experimental determination of the starting structure (PDB ID: 6VSB). ${ }^{10}$ It was shown that $\mathrm{pH}$ acidification of the medium does not associate with fusogenic conformational changes of some beta hCoV S proteins. ${ }^{27}$

The AScore/ShapeDock protocol from the ArgusLab 4.0.1 suite of programs ${ }^{28}$ was used for docking experiments. AScore takes into account the van der Waals interaction between the ligand and the protein, the hydrophobic effect, the hydrogen bonding between the ligand and the protein, the hydrogen bonding that involves charged donor and/or acceptor groups, the deformation effect and the effects of the translational and rotational entropy loss in binding process, respectively. The reproducible approach explores all energy minima during conformational searches. Its consistency for the flexible docking of ligands in the crystal structures of viral proteins was demonstrated ${ }^{29-33}$ by generating well-correlated binding free energies with experimental inhibitory concentrations. ${ }^{31}$ Also, a predicted dissociation constant of $48.7 \mu \mathrm{M}$ for the hCoV-OC43 S/9-O-Ac-Sia complex ${ }^{16}$ was in agreement with an experimental value of $49.7 \pm 10.7 \mu \mathrm{M}^{27}$

\section{RESULTS AND DISCUSSION}

A possible infection mechanism is based on dual host receptor recognition - the recognition of ACE2 by the RBD of SARS-CoV-2 S that is simultaneously supported by the recognition of the ganglioside-rich domain of the plasma membrane by the GBD of SARS-CoV-2 S. The GBD subdomain of the NTD of SARS-CoV-2 S consists of amino acids being located between 111 and 162 in the protein sequence and forming a functional 
domain that is entirely conserved in all clinical isolates. $^{3}$ The particular residues are mainly arranged in three loops, L1 (111-Asp-Ser-Lys-ThrGlu-Ser-116), L2 (121-Asn-Asn-Ala-Thr-Asn-Val126) and L3 (136-Cys-Asn-Asp-Pro-139), and in five hydrophobic pockets, P1 (Leu117, Leu118, Ile119 and Val120), P2 (Val126, Val127, Ile128, Lys129, Val130 and Cys131), P3 (Phe133, Val159 and Tyr160), P4 (Phe135 and Cys136) and P5 (Phe140, Leu141 and Phe157), as shown in Fig. 1 (bottom). To mimic the interaction between the SARS-CoV-2 transmembrane S glycoprotein and the ganglioside-rich domain, the interaction between GBD and 9-O-Ac-Sia was simulated by means of molecular docking (Fig. 2, top, right). The complex formed illustrates that Val159 from the P3 (Phe133, Val159 and Tyr160) hydrophobic pocket accommodates the 5-N-Ac methyl, Tyr160 from the same hydrophobic pocket accommodates the 9-O-Ac methyl, while Phe135 from the P4 (Phe135 and Cys136) hydrophobic pocket accommodates the 2-O methyl (Fig. 2, center). The complex is also stabilized by 9 electrostatic contacts of 9-O-Ac-Sia with the GBD residues (Fig. 2, bottom) - 2 between the 1-O hydroxyl and
Asp111 (2.97 and 2.84 $\AA$ ), 1 between the 2-O and Asp111 (3.08 $\AA), 1$ between the O6 and Asp111 $(2.63 \AA), 1$ between the 7-O and Asp111 (2.82 $\AA$ ), 1 between the 1-carboxylate and Cys136 (2.97 $\AA)$, 1 between the 5-N-Ac and Cys136 (2.24 $\AA$ ) and 2 between the 9-O-Ac and Ser161 (2.71 and 3.00 $\AA$ ), respectively. Thus, the particular sequence AspAspAspAspAspCysCysSerSer is the signature of the high specificity of 9-O-Ac-Sia to the GBD. Asp111, Phe135, Cys136, Tyr160 and Ser161 were previously identified using a molecular dynamics (MD) approach as the sites of SARS-CoV-2 S in contact with GM1 (a ganglioside with sialic acid), of which Asp111 contributed most to the stability of the NTD/9-O-Ac-Sia complex in a more realistic mode of MD simulation. ${ }^{3}$ Evaluated strength of the GBD/9-O-Ac-Sia interaction, seen through a dissociation constant of circa $17.5 \mu \mathrm{M}$ (Table 1), indicates potent S-mediated virion attachment, especially in high-density receptor environments. E.g., the GBD of SARS-CoV-2 S binds 9 -O-Ac-Sia $\left(\mathrm{K}_{\mathrm{d}} \approx 17.5 \mu \mathrm{M}\right)$ with about 3 times greater potency than does the interactive site of hCoV-OC43 S $\left(\mathrm{K}_{\mathrm{d}} \approx 48.7 \mu \mathrm{M}\right){ }^{16,27}$

Table 1

The binding free energies obtained by docking 9-O-Ac-Sia and Hcq in the GBD of the single point mutants of SARS-CoV-2 S

\begin{tabular}{|c|c|c|c|c|c|}
\hline \multicolumn{3}{|c|}{ Ligand: 9-O-Ac-Sia } & \multicolumn{3}{|c|}{ Ligand: Heq } \\
\hline Receptor $^{(\mathbf{a})}$ & $\Delta G_{\text {bind }}\left(\mathrm{kcal} \mathrm{mol}^{-1}\right)$ & $K_{d}(\mu M)^{(b)}$ & Receptor $^{(a)}$ & $\Delta G_{\text {bind }}\left(\mathrm{kcal} \mathrm{mol}^{-1}\right)$ & $K_{d}(\mu M)^{(b)}$ \\
\hline wt SARS-CoV-2 S & -6.53 & 17.50 & wt SARS-CoV-2 S & -7.96 & 1.60 \\
\hline Leu117Met & -6.60 & 15.56 & Phe157Met & -7.98 & 1.54 \\
\hline Glu132Ala & -6.62 & 15.05 & Asp138Met & -7.99 & 1,51 \\
\hline Asp138Arg & -6.64 & 14.55 & Phe135Lys & -8.03 & 1.41 \\
\hline Phe157Met & -6.64 & 14.55 & Thr114Ptr & -8.17 & 1.12 \\
\hline Thr124Arg & -6.69 & 13.38 & Asn121Leu & -8.17 & 1.12 \\
\hline Thr114Ptr & -6.69 & 13.38 & Asn121Met & -8.17 & 1.12 \\
\hline Asn121Phe & -6.69 & 13.38 & Asn121Phe & -8.17 & 1.12 \\
\hline Asn122Met & -6.69 & 13.38 & Asn125Ile & -8.18 & 1.10 \\
\hline Ala123Gln & -6.69 & 13.38 & Asn125Met & -8.18 & 1.10 \\
\hline Ala123Glu & -6.69 & 13.38 & Asp111Gly & -8.19 & 1.08 \\
\hline Ala123Lys & -6.69 & 13.38 & Asn137Pro & -8.19 & 1.08 \\
\hline Ser161Tyr & -6.88 & 9.73 & Val159Trp & -8.19 & 1.08 \\
\hline Asp138Leu & -6.91 & 9.25 & Asn121Ile & -8.20 & 1.06 \\
\hline Asp138Met & -6.91 & 9.25 & Ala123Arg & -8.20 & 1.06 \\
\hline Asp138Trp & -6.97 & 8.36 & Ala123His & -8.20 & 1.06 \\
\hline Phel40Ser & -6.98 & 8.23 & Ala123Gln & -8.20 & 1.06 \\
\hline Phe135Ala & -7.06 & 7.19 & Ala123Glu & -8.20 & 1.06 \\
\hline Val159Trp & -7.11 & 6.61 & Ala123Lys & -8.20 & 1.06 \\
\hline Val130Lys & -7.13 & 6.40 & Ala123Ptr & -8.20 & 1.06 \\
\hline Ile119Met & -7.17 & 5.98 & Ala123Tyr & -8.20 & 1.06 \\
\hline Asn121Leu & -7.17 & 5.98 & Thr124Arg & -8.20 & 1.06 \\
\hline Asn121Ile & -7.17 & 5.98 & Ile119Met & -8.21 & 1.05 \\
\hline Asn121Met & -7.17 & 5.98 & Asn122Ile & -8.21 & 1.05 \\
\hline Ala123Arg & -7.17 & 5.98 & Asn122Met & -8.21 & 1.05 \\
\hline Ala123His & -7.17 & 5.98 & Asn122Sep & -8.21 & 1.05 \\
\hline Ala123Ptr & -7.17 & 5.98 & Asn137Нyp & -8.21 & 1.05 \\
\hline Asn125Ile & -7.17 & 5.98 & Asp138Arg & -8.23 & 1.01 \\
\hline Asn125Met & -7.17 & 5.98 & Asp138Ptr & -8.24 & 0.99 \\
\hline
\end{tabular}


Table 1 (continued)

\begin{tabular}{c|c|c|c|c|c}
\hline Asn122Ile & -7.18 & 5.88 & Phe140Ser & -8.39 & 0.77 \\
Asn122Leu & -7.18 & 5.88 & Asp138Trp & -8.49 & -8.51 \\
Asn137Hyp & -7.18 & 5.88 & Asp138Leu & -8.78 & 0.65 \\
Asp111Gly & -7.20 & 5.69 & Leu117Met & -8.84 & 0.40 \\
Asn122Sep & -7.20 & 5.69 & Asn122Leu & -8.88 & 0.36 \\
Asp138Ptr & -7.21 & 5.59 & Val130Lys & -8.90 & 0.34 \\
Val127Glu & -7.29 & 4.89 & Phe135Ala & -8.94 & 0.33 \\
Ala123Tyr & -7.31 & 4.73 & Val127Glu & -9.10 & 0.31 \\
Asn137Pro & -7.31 & 4.73 & Glu132Ala & -9.24 & 0.23 \\
Phe135Lys & -7.38 & 4.20 & Ser161Tyr & -9.67 & 0.19 \\
Asn122Trp & -7.40 & 4.07 & Asn122Trp & -9.76 & 0.09 \\
Val120Tyr & -7.42 & 3.93 & Val120Tyr & & 0.08 \\
\hline
\end{tabular}

a) wild-type (wt), Hyp - hydroxyl Pro, Ptr - phosphorylated Thr, Tpo - phosphorylated Tyr, Sep - phosphorylated Ser

b) $\Delta \mathrm{G}_{\text {bind }}$ - the binding free energy, $\mathrm{K}_{\mathrm{d}}$ - the dissociation constant, $\Delta \mathrm{G}_{\text {bind }}=\mathrm{RT} \ln \left(\mathrm{K}_{\mathrm{d}}\right), \mathrm{R}-$ the gas constant $\left(1.9872 \times 10^{-3} \mathrm{kcal} \mathrm{K}\right.$ $\left.\mathrm{mol}^{-1}\right), \mathrm{T}$ - the absolute temperature $(300 \mathrm{~K}), 1 \mu \mathrm{M}=10^{-6} \mathrm{M}$

Each of the GBD residues is mutated to the other 24 (20 standard amino acids, phosphorylated Tyr, Ser and Thr, as well as hydroxyl Proline). All the single point mutants are energetically evaluated with reference to the original receptor (PDB ID: 6VSB). SPMs that stabilize the wt receptor structure for more than $2 \mathrm{kcal} \mathrm{mol}^{-1}$ are treated as likely ones (Table 1). An average level of thermochemical accuracy of $2 \mathrm{kcal} \mathrm{mol}^{-1}$ is acceptable for the structure-based drug (or ligand) design purposes. ${ }^{34-36}$

An inspection of the values in Table 1 shows that Asn122Trp and Val120Tyr stabilize the wt/9O-Ac-Sia complex to a largest extent. Relative to the original complex (Fig. 2, top, right), the particular SPMs do not change very much the overall spatial orientation of 9-O-Ac-Sia with respect to the receptor, but rather cause subtle conformational changes that are hard to be noted by looking at the binding modes superficially. Only the Val120Tyr/9-O-Ac-Sia interaction mode (Fig. 3) is characterized hereafter, considering that a similar, preliminary discussion on the Asn122Trp/9-O-Ac-Sia interaction mode is available online. ${ }^{37}$

Val120Tyr increases the hydrophobic nature of the GBD/9-O-Ac-Sia interaction. Val159 from the P3 (Phe133, Val159 and Tyr160) hydrophobic pocket accommodates the 5-N-Ac methyl, while Phe135 from the P4 (Phe135 and Cys136) hydrophobic pocket accommodates the 2-O methyl (Fig. 3, center), as similarly displayed by the wt/9O-Ac-Sia interaction interface (Fig. 2, center). The increased hydrophobicity is reflected through the interaction of the 9-O-Ac methyl not only with Tyr160 (Fig. 2, center), but with both Phe133 and
Tyr160 (Fig. 3, center). These are expected consequences of the particular SPM, considering the physicochemical features of the original amino acid and its substituent. Val120 - a small, aliphatic and hydrophobic amino acid is replaced by Tyr 120 - a large and hydrophobic amino acid having an aromatic ring in its side chain. As a hydrophobic amino acid does not like to reside in an aqueous environment, the SPM in the GBD increases the hydrophobic effect and the release of proteinbound water molecules, thereby causing to have the substituent (Tyr120) buried within the hydrophobic core of the NTD of SARS-CoV-2 S, or within the lipid portion of the membrane.

The introduction of Tyr120, instead of Val120, in the GBD is associated with 10 electrostatic contacts of 9-O-Ac-Sia with the GBD residues (Fig. 3, bottom) - 2 between the 1-O hydroxyl and Asp111 (3.19 and $2.88 \AA$ ), 1 between the 2-O and Asp111 (2.96 $\AA), 1$ between the O6 and Asp111 (2.73 $\AA), 1$ between the 7-O and Asp111 (2.94 $\AA$ ), 1 between the $5-\mathrm{N}-\mathrm{Ac}$ and $\mathrm{G} \ln 134$ (3.05 $\AA$ ), 1 between the 1-carboxylate and Cys136 (2.89 $\AA), 1$ between the 5-N-Ac and Cys136 (2.45 $\AA$ ) and 2 between the 9-O-Ac and Ser161 (2.89 and $3.48 \AA$ ), respectively. Thus, Val120Tyr changes the specificity pattern, AspAspAspAspAspCysCysSerSer, of the wt/9-OAc-Sia complex to AspAspAspAspAspGlnCysCysSerSer. The specificity order, extended by a Gln due to the Val120Tyr mutation, is simultaneously followed by the increased hydrophobic effect. 

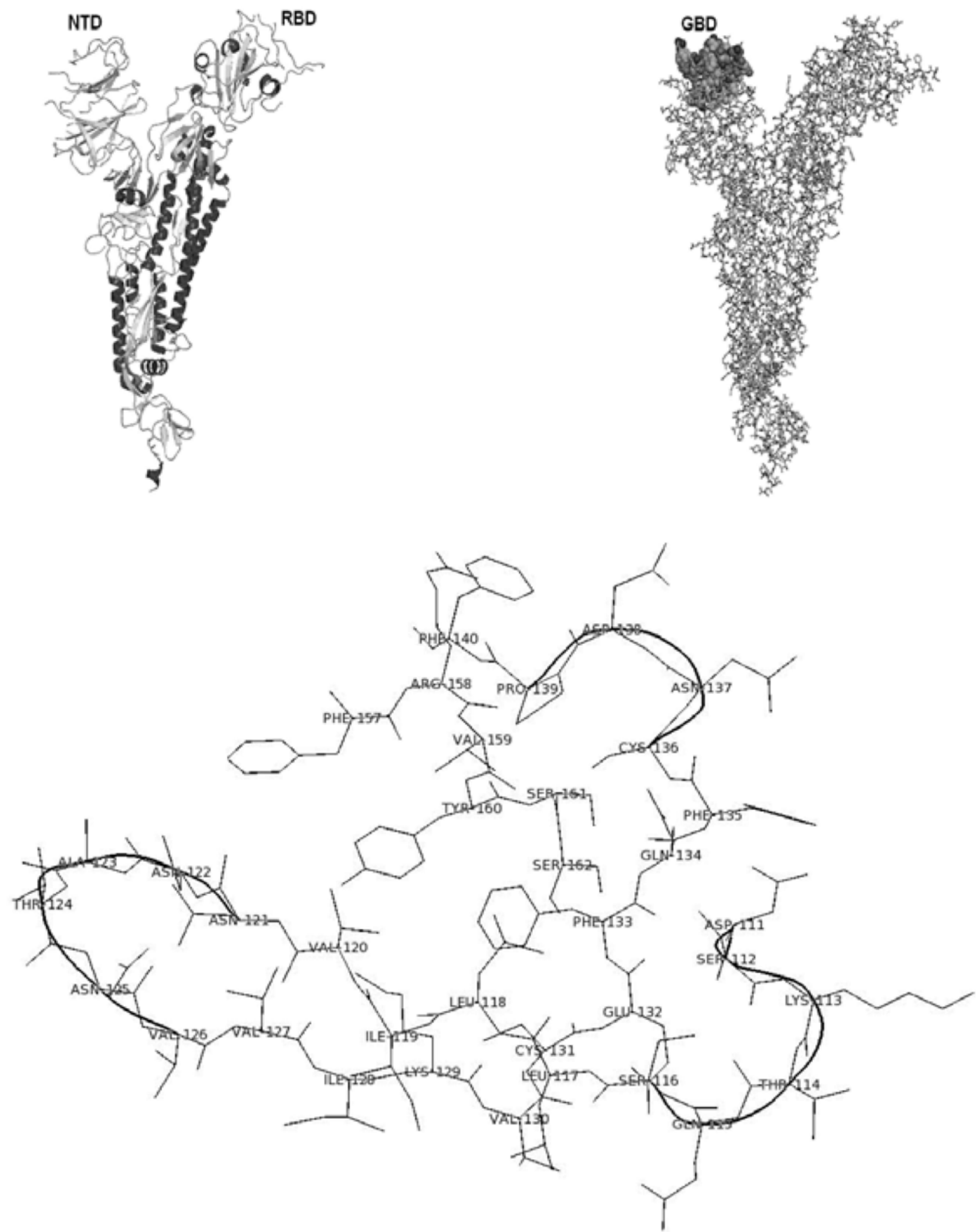

Fig. 1 - (top, left) The SARS-CoV-2 S glycoprotein (PDB ID: 6VSB) with two distinct domains - RBD and NTD; (top, right) the NTD residues being between 111 and 162 (denoted by spheres) in the protein sequence form GBD; (bottom) GBD consists of three loops (denoted by ribbon), L1 (111-Asp-Ser-Lys-Thr-Glu-Ser-116), L2 (121-Asn-Asn-Ala-Thr-Asn-Val-126) and L3 (136-Cys-AsnAsp-Pro-139), and five hydrophobic pockets, P1 (Leu117, Leu118, Ile119 and Val120), P2 (Val126, Val127, Ile128, Lys129, Val130 and Cys131), P3 (Phe133, Val159 and Tyr160), P4 (Phe135 and Cys136) and P5 (Phe140, Leu141 and Phe157). 


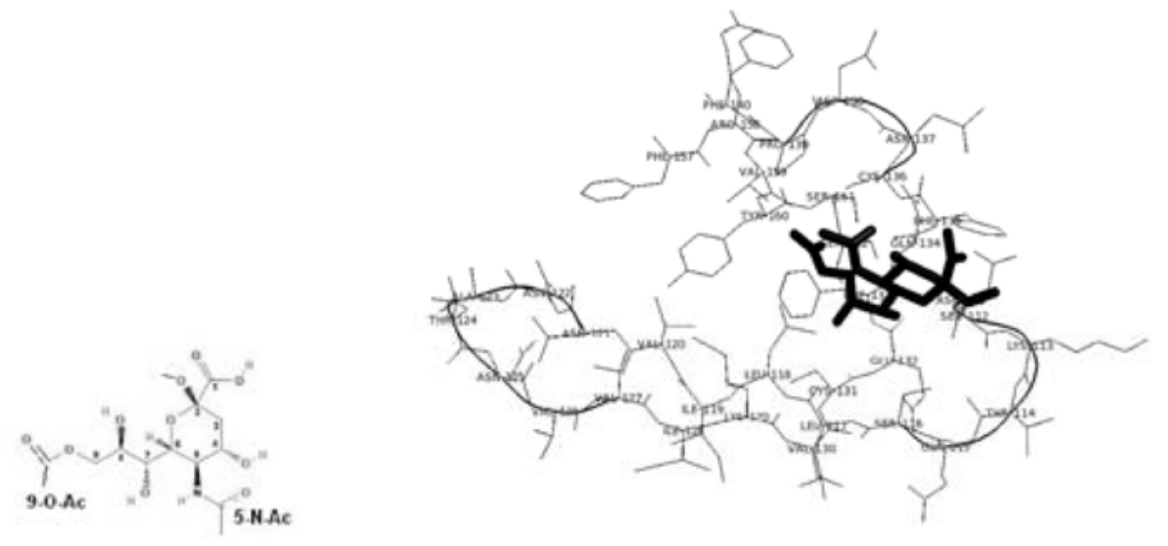

9-0-Ac-Sia
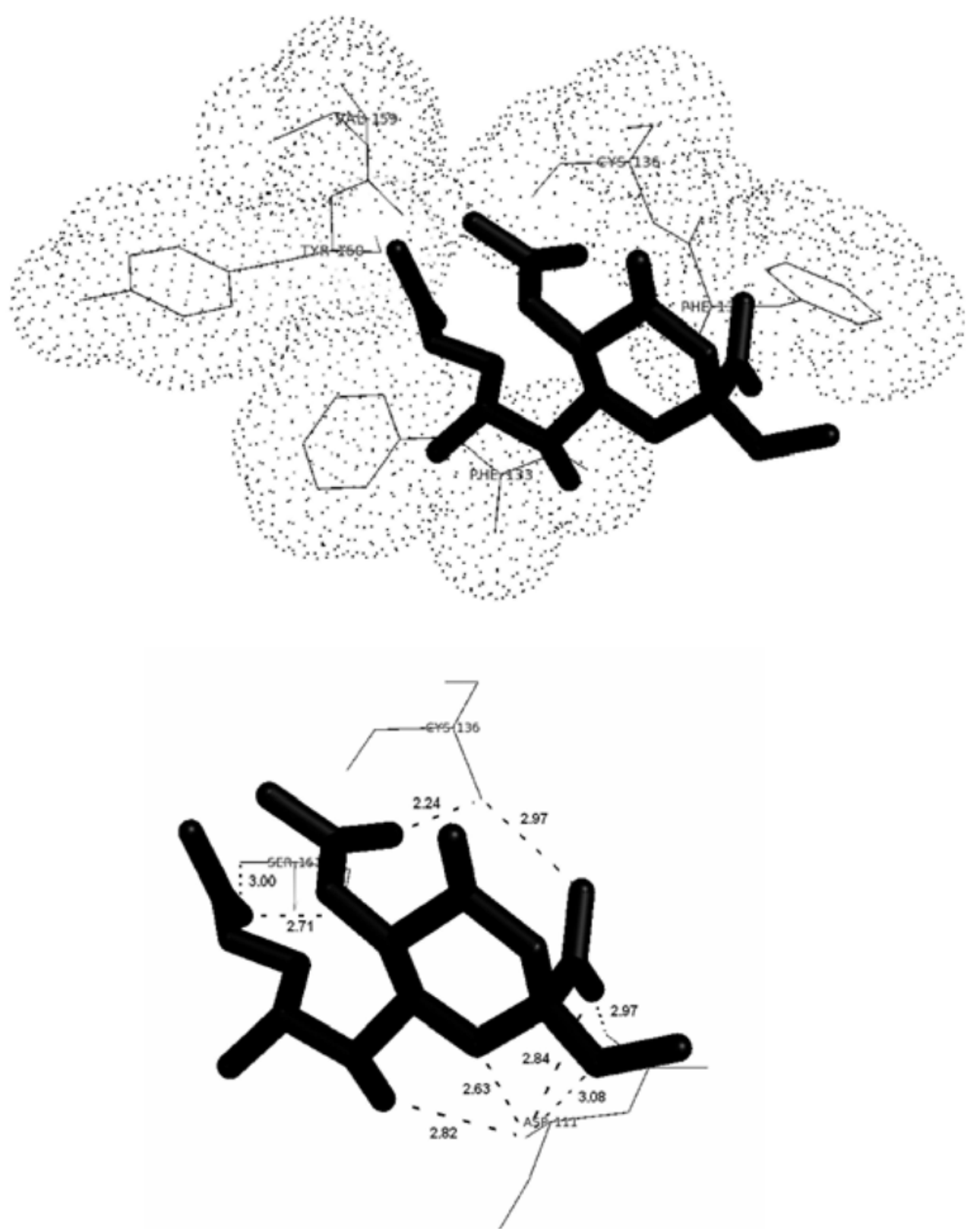

Fig. 2 - (top, left) 9-O-Ac-Sia structure; (top, right) 9-O-Ac-Sia (bold sticks) docked in GBD; (center) Val159 (dots) accommodates the 5-N-Ac methyl, Tyr160 (dots) accommodates the 9-O-Ac methyl, and Phe135 accommodates the 2-O methyl; (bottom) 9 electrostatic contacts of 9-O-Ac-Sia with GBD residues - 5 with Asp111, 2 with Cys136 and 2 with Ser161, respectively. 

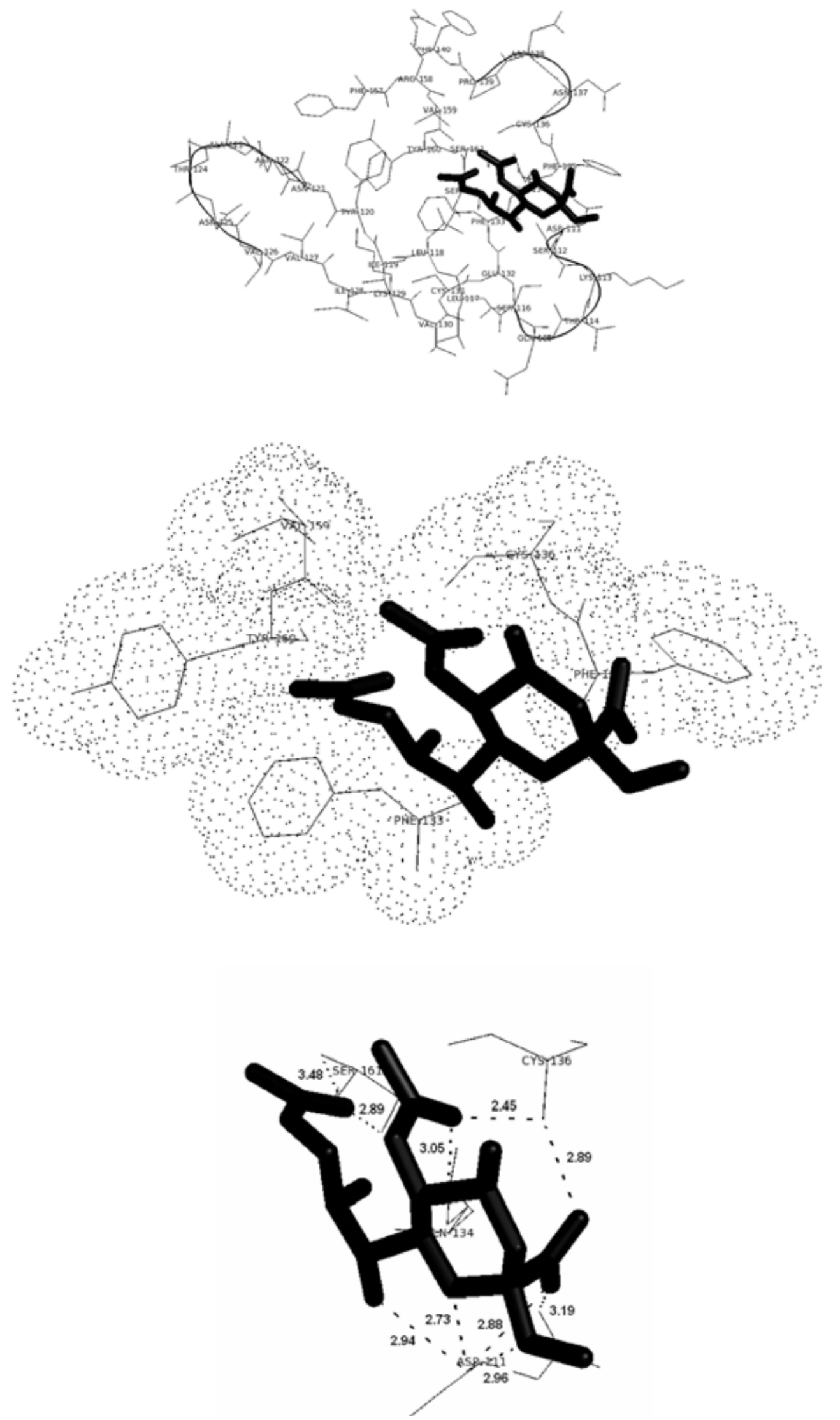

Fig. 3 - (top) 9-O-Ac-Sia (bold sticks) docked in the Val120Tyr GBD; (center) Val159 (dots) accommodates the 5-N-Ac methyl, Phe133 and Tyr160 (dots) accommodate the 9-O-Ac methyl, and Phe135 accommodates the 2-O methyl; (bottom) 10 electrostatic contacts of 9-O-Ac-Sia with GBD residues - 5 with Asp111, 1 with Gln134, 2 with Cys136 and 2 with Ser161, respectively. 

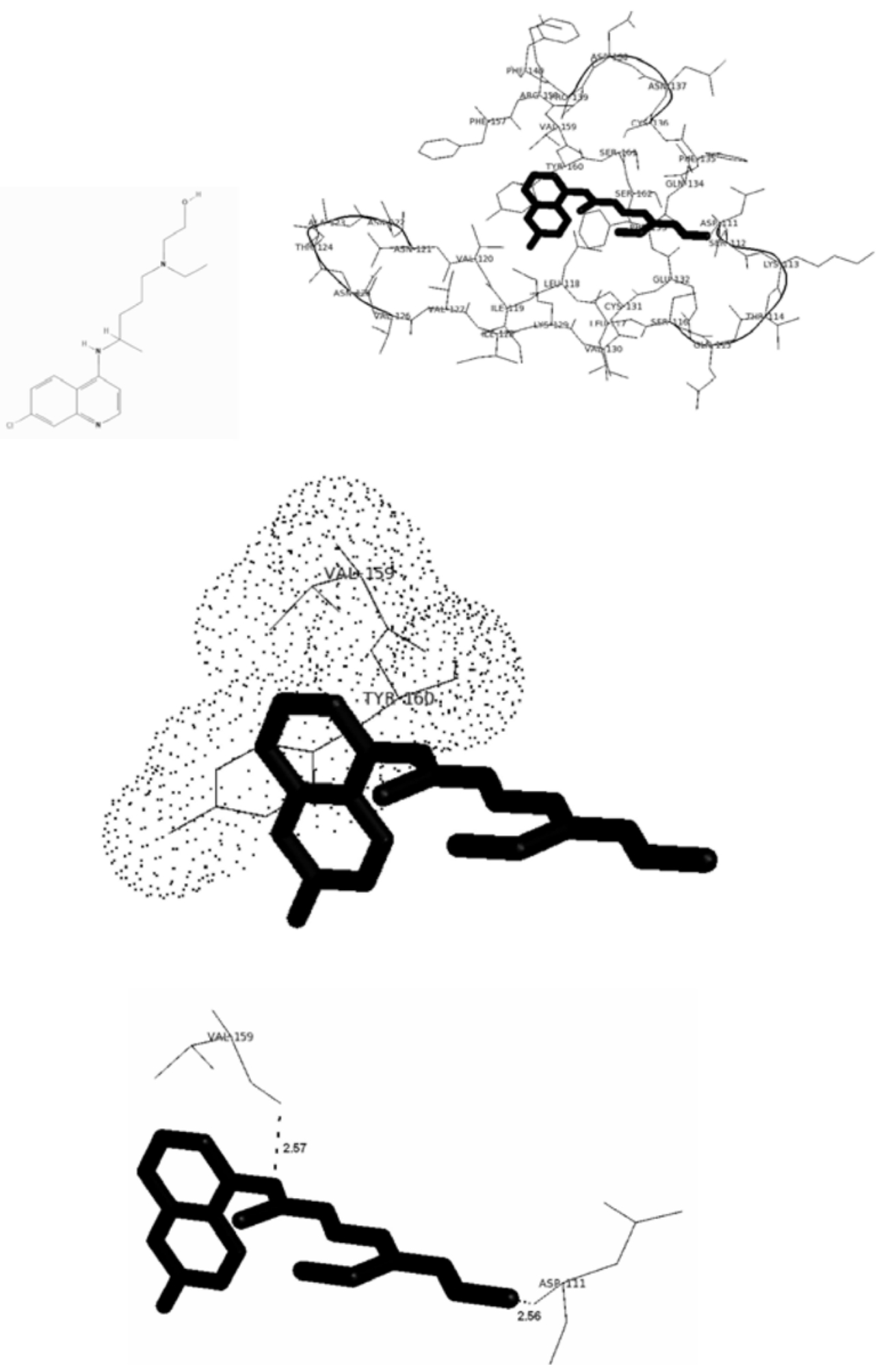

Fig. 4 - (top, left) Hcq structure; (top, right) Hcq (bold sticks) docked in GBD; (center) Val159 and Tyr160 (dots) accommodate the aromatic core scaffold of Hcq; (bottom) 2 electrostatic contacts of the Hcq side chain with GBD residues - 1 with Asp111 and 1 with Val159, respectively.

The chemical structure of Hcq (Fig. 4, top, left) contains an aromatic core to which both a $\mathrm{Cl}$ atom and a large side chain are bound, indicating a pronounced hydrophobic character of the ligand.
The binding mode obtained by docking Hcq in the GBD of SARS-CoV-2 $\mathrm{S}$ is illustrated in Fig. 4 (top, right). Val159 and Tyr160 from the P3 (Phe133, Val159 and Tyr160) hydrophobic pocket 
accommodate the aromatic core scaffold of Hcq (Fig. 4, center). Two electrostatic contacts between the Hcq side chain and the GBD residues are also involved in formation of the complex - 1 with Asp111 (2.56 $\AA)$ and 1 with Val159 (2.57 $\AA)$, respectively (Fig. 4, bottom).

The values in Table 1 indicate that Asn122Trp and Val120Tyr are the most likely SPMs for the binding of Hcq. The introduction of Tyr120 (Fig. 5, top), instead of Val120 (Fig. 4, top, right), in the GBD causes the vertical conformational switch (rotation by around $270^{\circ}$ clockwise) of Hcq. The orientation of the $\mathrm{Cl}$ atom being right toward Tyr160 additionally contributes to the much more hydrophobic nature of the Val120Tyr/Hcq interaction compared to the $\mathrm{wt} / \mathrm{Hcq}$ interaction. Leu118 and Tyr120 (dots) from the P1 (Leu117, Leu118, Ile119 and Tyr120) hydrophobic pocket and the entire P3 (Phe133, Val159 and Tyr160) hydrophobic pocket (dots) accommodate the aromatic core scaffold of Hcq (Fig. 5, bottom). One electrostatic contact between the Hcq side chain and Cys136 (3.03 A, Fig. 5, bottom) underlines the remarkably low specificity of Hcq binding. The hydrophobic nature of the Val120Tyr/Hcq interaction mode (Fig. 5) is qualitatively comparable to that of the Asn122Trp/Hcq interaction mode. ${ }^{37}$
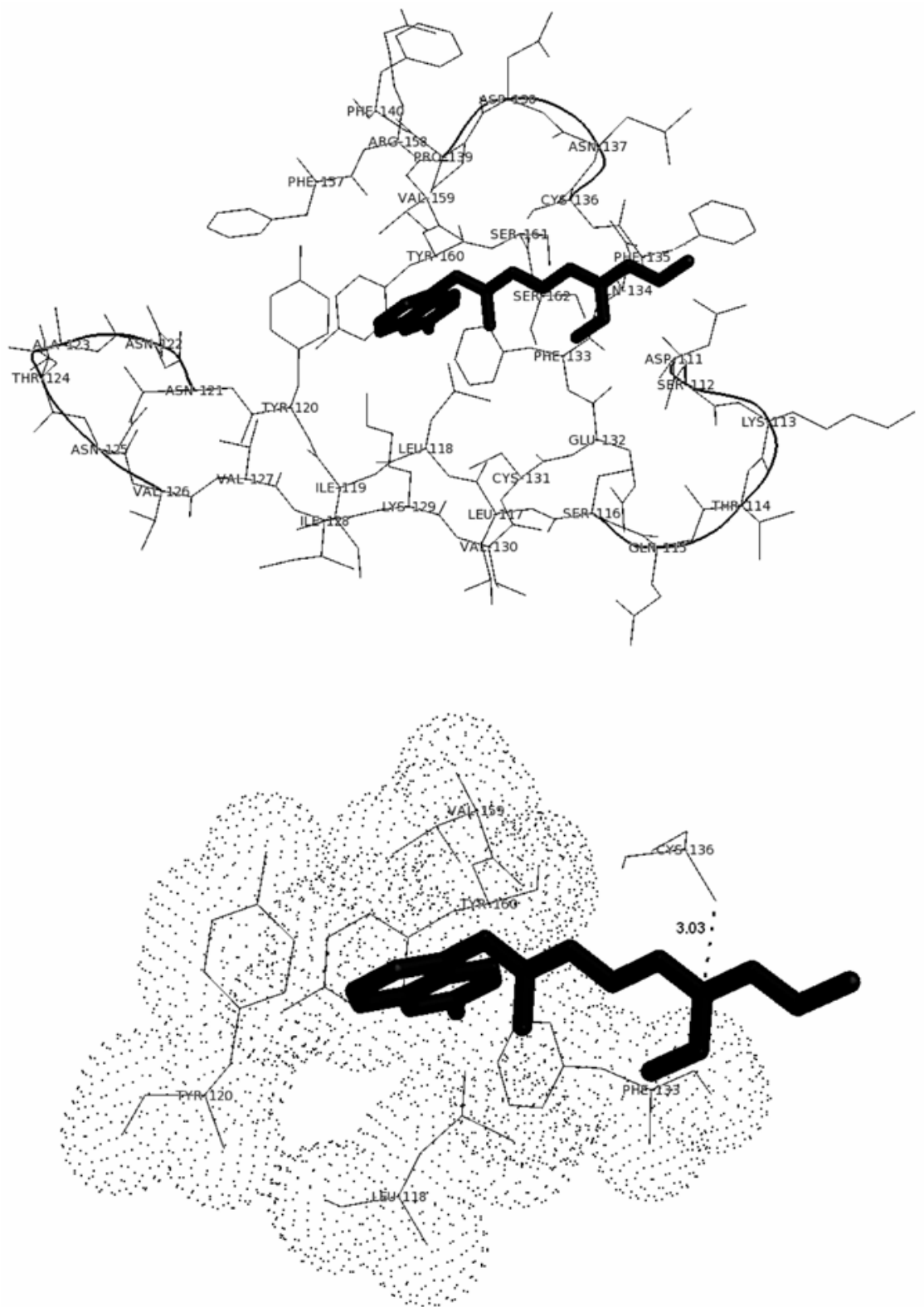

Fig. 5 - (top) Hcq (bold sticks) docked in the Val120Tyr GBD; (bottom) Leu118 and Tyr120 (dots) and the entire P3 (Phe133, Val159 and Tyr160) hydrophobic pocket (dots) accommodate the aromatic core scaffold of Hcq; 1 electrostatic contact of the Hcq side chain with Cys136. 
The binding free energies (Table 1) of the complexes, formed by docking 9-O-Ac-Sia and $\mathrm{Hcq}$ to the same receptor, show that $\mathrm{Hcq}$ has higher affinity to SARS-CoV-2 $\mathrm{S}$ than does 9-OAc-Sia. This standpoint can be rationalized by the considerably hydrophobic and almost non-specific recognition of SARS-CoV-2 S by Hcq, thereby speaking in favor of the potential of Heq to competitively inhibit the interactions of GBD with lipid rafts. However, rapid kinetics underlying Smediated virion attachment to 9-O-Ac-Sia is associated with recognition that is very specific. It is, therefore, convenient to contrast the chemical structures of 9-O-Ac-Sia and Hcq by estimating their respective entropy losses upon binding. Flexible ligand docking is based on active torsions in ligand structure, conceivable as particular $\mathrm{sp}^{3}$ bonds that are directly involved in finding the lowest energy receptor/ligand conformations. Entropy loss upon ligand binding is related to the loss of its degrees of freedom. The torsional potential indirectly takes care of the particular entropy amount by being proportional to the number of active torsions in ligand structure. An active torsion has been energetically estimated to cost circa $0.3 \mathrm{kcal} \mathrm{mol}^{-1} .^{38,39}$ It means that the structure of 9-O-Ac-Sia with eleven active torsions experiences a negative entropy change of $-3.3 \mathrm{kcal}$ $\mathrm{mol}^{-1}$, while the structure of Hcq with one active torsion experiences a tiny entropic decrease of -0.3

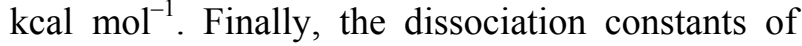
the SARS-CoV-2 S/Hcq complexes are in the micromolar $\left(10^{-6} \mathrm{M}\right)$ range (Table 1$)$, which is acceptable for hit ligand molecule but not for drug candidate.

\section{CONCLUSIONS}

Val120Tyr and Asn122Trp are predicted as the most likely SPMs in the GBD of SARS-CoV-2 S involved in very specific recognition with 9-O-AcSia through electrostatic interactions. The same SPMs are found to be critical in the GBD of SARS-CoV-2 $\mathrm{S}$ involved in very hydrophobic recognition with Hcq, thereby indicating the ability of Hcq to competitively inhibit GBD interactions with lipid rafts. However, the considerably nonspecific binding of $\mathrm{Hcq}$ and the micromolar range of the dissociation constants of the SARS-CoV-2 $\mathrm{S} / \mathrm{Hcq}$ complexes do not support the proposal of treating Hcq as a drug candidate. Keeping a clear structural similarity of a potential drug candidate with a natural substrate, accompanied by essential functional group modifications, may be a reasonable guideline for the structure-based design of anti-CoViD-19 drugs. A relevant example may be zanamivir (Relenza), an anti-influenza drug that was directly derived from sialic acid with minimal functional modifications. ${ }^{40,41}$

\section{REFERENCES}

1. J. Reguera, G. Mudgal, C. Santiago and J. M. Casasnovas, Virus Res., 2014, 194, 3-15.

2. D. S. Hui, E. I. Azhar, T. A. Madani, F. Ntoumi, R. Kock, O. Dar, G. Ippolito, T. D. Mchugh, Z. A. Memish, C. Drosten, A. Zumla and E. Petersen, Int. J. Infect. Dis., 2020, 91, 264-266.

3. J. Fantini, C. Di Scala, H. Chahinian and N. Yahi, Int. J. Antimicrob. Agents, 2020, 55, 105960.

4. J. Liu, R. Cao, M. Xu, X. Wang, H. Zhang, H. Hu, Y. Li, Z. Hu, W. Zhong and M. Wang, Cell Discovery, 2020, 6, 16.

5. R. R. Narkhede, R. S. Cheke, J. P. Ambhore and S. D. Shinde, EJMO, 2020, 4, 185-195.

6. N. A. Vaidya and R. Vyas, Front. Pharmacol., 2020, 11, 569665.

7. D. P. Misra, A. Y. Gasparyan and O. Zimba, Rheumatol. Int., 2020, 40, 1741-1751.

8. R. Kumar, A. Sharma, J. K. Srivastava, M. H. Siddiqui, M. S. Uddin and L. Aleya, Environ. Sci. Pollut. Res. Int., 2021, 15, 1-14.

9. D. R. Boulware, M. F. Pullen, A. S. Bangdiwala, K. A. Pastick, S. M. Lofgren, E. C. Okafor, C. P. Skipper, A. A. Nascene, M. R. Nicol, M. Abassi, N. W. Engen, M. P. Cheng, D. LaBar, S. A. Lother, L. J. MacKenzie, G. Drobot, N. Marten, R. Zarychanski, L. E. Kelly, I. S. Schwartz, E. G. McDonald, R. Rajasingham, T. C. Lee and K. H. Hullsiek, N. Engl. J. Med., 2020, 383, 517-525.

10. D. Wrapp, N. Wang, K. S. Corbett, J. A. Goldsmith, C. L. Hsieh, O. Abiona, B. S. Graham and J. S. McLellan, Science, 2020, 367, 1260-1263.

11. H. Yao, X. Lu, Q. Chen, K. Xu, Y. Chen, L. Cheng, F. Liu, Z. Wu, H. Wu, C. Jin, M. Zheng, N. Wu, C. Jiang and L. Li, medRxiv, 2020, DOI: 10.1101/2020.04.14.20060160.

12. B. Korber, W. M. Fischer, S. Gnanakaran, H. Yoon, J. Theiler, W. Abfalterer, B. Foley, E. E. Giorgi, T. Bhattacharya, M. D. Parker, D. G. Partridge, C. M. Evans, T. M. Freeman, T. I. de Silva and A. Angyal, bioRxiv, 2020, DOI: 10.1101/2020.04.29.069054.

13. P. M. Mitrasinovic, Curr. Drug Targets, 2013, 14, 817-829.

14. P. M. Mitrasinovic, Med. Chem., 2014, 10, 252-270.

15. P. M. Mitrasinovic, Med. Chem., 2014, 10, 46-58.

16. P. M. Mitrasinovic, Acta Chim. Slov., 2020, 67, 949-956.

17. P. Vanhee, E. Verschueren, L. Baeten, F. Stricher, L. Serrano, F. Rousseau and J. Schymkowitz, Nucleic Acids Res., 2011, 39(suppl1), D435-D442.

18. M. Petukhov, D. Cregut, C. M. Soares and L. Serrano, Protein Sci., 1999, 8, 1982-1989.

19. V. Muñoz and L. Serrano, Proteins, 1994, 20, 301-311.

20. R. Abagyan and M. Totrov, J. Mol. Biol., 1994, 235, 983-1002. 
21. M. Vijayakumar, K. Y. Wong, G. Schreiber, A. R. Fersht, A. Szabo and H. X. Zhou, J. Mol. Biol., 1998, 278, 10151024.

22. J. W. H. Schymkowitz, F. Rousseau, I. C. Martins, J. Ferkinghoff-Borg, F. Stricher and L. Serrano, Proc. Natl. Acad. Sci. U. S. A., 2005, 102, 10147-10152.

23. J. Van Durme, J. Delgado, F. Stricher, L. Serrano, J. Schymkowitz and F. Rousseau, Bioinformatics, 2011, 27, 1711-1712.

24. J. Schymkowitz, J. Borg, F. Stricher, R Nys, F. Rousseau and L. Serrano, Nucleic Acids Res., 2005, 33(suppl2), W382-W388.

25. L. Baeten, J. Reumers, V. Tur, F. Stricher, T. Lenaerts, L. Serrano, F. Rousseau and J. Schymkowitz, PLoS Comput. Biol., 2008, 4, e1000083.

26. R. Guerois, J. E. Nielsen and L. Serrano, J. Mol. Biol., 2002, 320, 369-387.

27. M. Alejandra Tortorici, A. C. Walls, Y. Lang, C. Wang, Z. Li, D. Koerhuis, G.-J. Boons, B.-J. Bosch, F. A. Rey, R. J. de Groot and D. Veesler, Nat. Struct. \& Mol. Biol., 2019, 26, 481-489.

28. M. A. Thompson, "ArgusLab 4.0.1", Planaria Software, Seattle, 2004.

29. P. M. Mitrasinovic, Biophys. Chem., 2009, 140, 35-38.

30. P. M. Mitrasinovic, Curr. Drug Targets, 2010, 11, 315-326.
31. M. L. Mihajlovic and P. M. Mitrasinovic, Mol. Simulation, 2009, 35, 311-324.

32. M. L. Mihajlovic and P. M. Mitrasinovic, Biophys. Chem., 2008, 136, 152-158.

33. M. L. Mihajlovic and P. M. Mitrasinovic, J. Serb. Chem. Soc., 2009, 74, 1-13.

34. A. D. Becke, J. Chem. Phys., 2002, 117, 6935-6938.

35. S. Cosconati, S. Forli, A. L. Perryman, R. Harris, D. S. Goodsell and A. J. Olson, Expert Opin. Drug Discov., 2010, 5, 597-607.

36. N. Deng, L. Wickstrom, P. Cieplak, C. Lin and D. Yang, J. Phys. Chem. B, 2017, 121, 10484-10497.

37. P. M. Mitrasinovic, ChemRxiv, 2020, DOI: 10.26434/chemrxiv.12765953.v1.

38. G. M. Morris, D. S. Goodsell, R. S. Halliday, R. Huey, W. E. Hart, R. K. Belew and A. J. Olson, J. Comput. Chem., 1998, 19, 1639-1662.

39. G. M. Morris, R. Huey, W. Lindstrom, M. F. Sanner, R. K. Belew, D. S. Goodsell and A. J. Olson, J. Comput. Chem., 2009, 30, 2785-2791.

40. M. Von Itzstein, Nat. Rev. Drug Discov., 2007, 6, 967974.

41. P. M. Mitrasinovic, "Global View of the Fight against Influenza", Nova Science Publishers, New York, 2009, p. 185-192. 
